\title{
Diagnóstico prenatal de anomalías nefrourológicas en el Centro de Referencia Perinatal Oriente, Santiago, Chile
}

\section{Prenatal diagnosis of nefrourological anomalies at the Eastern Perinatal Referral Center, Santiago, Chile}

\author{
María P. Marín ${ }^{1 *}$, María C. Sierralta ${ }^{2}$, Paulina Ortega ${ }^{3}$, Daniel Martín ${ }^{2}$, Sergio de la Fuente ${ }^{2}$ y \\ Juan G. Rodríguez²
}

${ }^{1}$ Programa de Especialización en Medicina Materno Fetal; ${ }^{2}$ Centro de Referencia Perinatal Oriente (CERPO); ${ }^{3}$ Programa Especialización Ginecología y Obstetricia. Facultad de Medicina, Universidad de Chile, Santiago, Chile

\section{Resumen}

Introducción: Las anomalías congénitas de los riñones y las vías urinarias (CAKUT, Congenital Anomalies of the Kidney and Urinary Tract) representan un 20-30\% de las anomalías detectadas en el periodo prenatal. Si bien la mayoría son de buen pronóstico, un $25 \%$ se asocian a enfermedad renal crónica en la infancia y en los casos graves a mortalidad perinatal. Objetivo: Describir los casos ingresados al Centro de Referencia Perinatal Oriente (CERPO) y determinar los resultados perinatales y la sobrevida al año. Método: Estudio descriptivo y retrospectivo. Se incluyeron pacientes ingresadas en la base de datos CERPO, entre los años 2003 y 2019, con diagnóstico de anomalía nefrourológica. Se incluyeron antecedentes prenatales, perinatales y de seguimiento posnatal al año. Resultados: Se evaluaron 273 pacientes. La edad gestacional promedio de derivación fue de 29 semanas + 2 días. El diagnóstico más frecuente fueron las anomalías del tracto de salida (69\%). Un 40\% de los casos se asociaron a otras anomalías congénitas, siendo las cardiopatías las más frecuentes (19\%). Aceptaron la realización de estudio genético invasivo 38 pacientes, de las cuales un $34 \%$ presentaron aneuploidías, siendo las trisomías 18 y 13 las más frecuentes (17\% y 6\%, respectivamente). Se instalaron cinco shunts derivativos vesicoamnióticos en fetos diagnosticados con megavejiga. La sobrevida global para la patología nefrourológica fue del $63 \%$ al año, y la mortalidad fetal y neonatal fue del $7 \%$ y el $16 \%$, respectivamente. La sobrevida al año según el grupo de clasificación CAKUT fue del 22\% para las anomalías de número de riñones, del $46 \%$ para las anomalías de tamaño y de morfología renal, del 60\% para las anomalías de la posición renal y del $72 \%$ para las anomalías del tracto de salida. En este último grupo, la sobrevida alcanza el $81 \%$ al excluir los pacientes con diagnóstico prenatal de megavejiga, que presentaron una sobrevida al año del $28 \%$. Los casos de patología nefrourológica asociada a oligohidramnios (índice de líquido amniótico $<5 \mathrm{~cm}$ ) sin evidencias de uropatía obstructiva asociada presentaron una sobrevida al año del 3\%. Conclusiones: Las anomalías del sistema nefrourológico son un diagnóstico prenatal frecuente. La sobrevida al año fue del $63 \%$, pero es necesario prolongar el seguimiento a largo plazo para determinar la evolución de la función renal en cada diagnóstico. La asociación de patología nefrourológica y oligohidramnios conlleva una sobrevida menor.

Palabras clave: CAKUT. Diagnóstico prenatal. Shunt vesicoamniotico. Pronóstico.

Correspondencia:

*María P. Marín

E-mail: mariapaz.marin@gmail.com

0048-766X / @ 2021 Sociedad Chilena de Obstetricia y Ginecología. Publicado por Permanyer. Éste es un artículo open access bajo la licencia CC BY-NC-ND (https://creativecommons.org/licenses/by-nc-nd/4.0/).
Disponible en internet: 27-10-2021 Rev Chil Obstet Ginecol. 2021;86(4):390-396 www. rechog.com 


\section{Abstract}

Introduction: Congenital anomalies of the kidneys and urinary tract (CAKUT) represent 20 to $30 \%$ of the anomalies detected prenatally. Although most are of good prognosis, $25 \%$ are associated with chronic kidney disease in childhood and severe cases with perinatal mortality. Objective: To describe the cases studied in the Eastern Perinatal Referral Center (CERPO) and to determine the perinatal outcome and survival at one year. Method: Descriptive and retrospective study. Patients registered on the CERPO database, between 2003 to 2019, with diagnosis of nephrourological anomaly were included. Antenatal, perinatal and postnatal follow-up information was collected. Results: 273 patients were evaluated. The average gestational age at referral was $29+2$ weeks. The main diagnosis was urinary tract outlet disorders (69\%). Thirty nine percent of the cases were associated with other congenital anomalies, with heart disease being the most frequent (19\%). Thirty-eight patients accepted an invasive procedure for genetic study, 34\% presented aneuploidy, trisomy 18 and 13 were the most frequent (17\% and $6 \%$ respectively). Five vesico-amniotic derivative shunts were installed in fetuses diagnosed with megabladder. Overall survival at one year was $63 \%$ and fetal and neonatal mortality were $7 \%$ and $16 \%$ respectively. One-year survival per group according to CAKUT classification was $22 \%$ in kidney number abnormalities, $46 \%$ in kidney size and morphology abnormalities, $60 \%$ in renal position abnormalities and $72 \%$ in outflow tract abnormalities. In the latter, survival reaches $81 \%$ excluding the patients with prenatal diagnosis of megabladder who had a one-year survival of $28 \%$. The cases of nephrourological pathology associated with oligohydramnios (amniotic fluid index $<5 \mathrm{~cm}$ ) without evidence of associated obstructive uropathy presented a survival of $3 \%$ at one year. Conclusions: The anomalies of the nephrourological system correspond to a frequent prenatal diagnosis. Overall, the one-year survival was $63 \%$; however, follow-up must continue to determine the evolution of renal function in relation to each diagnosis.

Key words: CAKUT. Prenatal diagnosis. Vesicoamniotic shunt. Prognosis.

\section{Introducción}

Las anomalías congénitas de los riñones y las vías urinarias (CAKUT, Congenital Anomalies of the Kidney and Urinary Tract) son trastornos que surgen durante el desarrollo embrionario o fetal y resultan en un espectro de defectos en los riñones y las vías urinarias que incluyen los uréteres, la vejiga y la uretra ${ }^{1}$. Estas representan un $20-30 \%$ de las anomalías diagnosticadas en el periodo prenatal ${ }^{2,3}$, pueden ser unilaterales o bilaterales, y acompañarse de anomalías en otros sistemas. Las malformaciones congénitas del riñón se definen macroscópicamente por cambios en el tamaño, la forma, la posición o el número de los riñones, o microscópicamente por un número reducido de nefronas o una histología anormal (Tabla 1) 1 .

Las alteraciones nefrourológicas congénitas son el origen de hasta un $50 \%$ de los casos de enfermedad renal crónica que requieren terapia de reemplazo renal en la infancia. Por lo anterior, es importante diagnosticar estas anomalías e iniciar la terapia precozmente para minimizar el daño renal, prevenir o retrasar la aparición de enfermedad terminal y manejar adecuadamente para evitar complicaciones de enfermedad renal crónica. Alrededor del $60 \%$ de los niños que se someten a cirugía por problemas renales 0 del tracto urinario en sus primeros 5 años de vida son identificados por ultrasonido prenatal ${ }^{4}$.
La mayoría de los casos presentan una dilatación leve de la pelvis renal, con buen resultado posnatal. Los casos clasificados como «uropatía obstructiva» tienen una dilatación bilateral más grave de la pelvis y la vejiga, secundaria a una obstrucción del tracto urinario inferior, típicamente valvas uretrales posteriores en los niños ${ }^{5}$. Además, los fetos con malformaciones que implican una reducción en el número o en el tamaño de los riñones tienen más probabilidades de tener un mal pronóstico renal ${ }^{6}$. Los riñones displásicos bilaterales pueden causar la secuencia de Potter, con muerte por hipoplasia pulmonar?. Para los sobrevivientes, son la causa más frecuente de enfermedad renal crónica en la infancia y representan el $25 \%$ de los niños que requieren terapia de reemplazo renal, según el registro del Reino Unido ${ }^{8}$.

La tasa global de anomalías nefrourológicas en recién nacidos vivos y mortinatos es de 0.3-1.6 por $1000^{9-11}$. La incidencia es mayor en pacientes con antecedentes familiares de patología renal y antecedentes maternos de enfermedad renal o diabetes mellitus. De todas las anomalías del espectro CAKUT diagnosticadas prenatalmente, la más frecuente es la dilatación del tracto urinario alto.

Las malformaciones renales se asocian con otras anomalías congénitas en aproximadamente el $30 \%$ de Ios casos ${ }^{11}$. La combinación de CAKUT y anomalías no renales se encuentra en más de 200 síndromes descritos $^{12}$. 
Rev Chil Obstet Ginecol. 2021;86(4)

Tabla 1. Categorias del espectro cakut

\begin{tabular}{|c|c|c|}
\hline Tipo de anomalía & Enfermedad & Definición \\
\hline $\mathrm{N}^{\circ}$ de riñones & Agenesia renal & Unilateral o bilateral, el riñón y el sistema de salida no se forman. \\
\hline Tamaño y morfología renal & $\begin{array}{l}\text { Hipoplasia renal } \\
\text { Displasia renal } \\
\text { Displasia renal multiquística }\end{array}$ & $\begin{array}{l}\text { Unilateral o bilateral, la forma del riñón es normal, pero de menor } \\
\text { tamaño y con menor número de nefronas. } \\
\text { Unilateral o bilateral, la forma del riñón y la diferenciación tisular son } \\
\text { anormales, con número reducido de nefronas. } \\
\text { Múltiples quistes dentro de un riñón displásico que le da una forma } \\
\text { anormal. }\end{array}$ \\
\hline Posición renal & $\begin{array}{l}\text { Riñón en herradura } \\
\text { Riñón ectópico/pélvico }\end{array}$ & $\begin{array}{l}\text { Los riñones se fusionan posteriormente con forma de herradura } \\
\text { Riñón en un ubicación anormal, típicamente pélvica }\end{array}$ \\
\hline Anomalías del tracto de salida & $\begin{array}{l}\text { Doble sistema colector } \\
\text { Megauréter } \\
\text { Válvas uretrales posteriores }\end{array}$ & $\begin{array}{l}\text { Unilateral o bilateral, la unión entre el riñón y el uréter está obstruida } \\
\text { lo que impide el drenaje de orina desde la pelvis del riñón } \\
\text { Unilateral o bilateral, la unión entre el uréter y la vejiga es defectuosa } \\
\text { lo que provoca un reflujo de orina desde la vejiga } \\
\text { Unilateral o bilateral, duplicación de uréter y pelvis renal, puede } \\
\text { acompañarse de riñones duplicados. El sistema de flujo de salida } \\
\text { puede presentar reflujo o presentar obstrucción } \\
\text { Distensión unilateral o bilateral del uréter que provoca defectos en el } \\
\text { flujo de orina. } \\
\text { Membrana que se forma en la uretra impidiendo el vaciado de la } \\
\text { vejiga, limitada a los hombres. }\end{array}$ \\
\hline
\end{tabular}

En Chile, el estudio ECLAMC ${ }^{13}$, realizado entre los años 1998 y 2010, encontró una incidencia de malformaciones urinarias de 64.5 por 10,000 nacimientos. La distribución por sexos demostró un significativo predominio masculino $(p<0.0001)$ y la hidroureteronefrosis fue la anomalía más frecuente ( $24.2 \%$ de los casos). Además, entre los factores de riesgo estudiados, el antecedente familiar de patología renal estuvo presente en el $32 \%$ de los casos.

El objetivo de este estudio fue caracterizar las anomalías renales y del tracto urinario evaluadas en el Centro de Referencia Perinatal Oriente (CERPO) y determinar los resultados neonatales en este grupo de pacientes.

\section{Método}

Se realizó un estudio descriptivo, retrospectivo, utilizando la base de datos de CERPO (Filemaker pro $7.0 \mathrm{v} 2$ ), que recopila la información de cada paciente de forma prospectiva. Este centro recibe pacientes derivadas desde atención primaria y otros hospitales, tanto de la Región Metropolitana (Servicio de Salud Metropolitano Oriente) como de otras regiones asignadas por la Red Pública de Salud, Santiago, Chile. Ante la sospecha de patología nefrourológica, se realiza una evaluación ecográfica y, en caso de confirmarse el diagnóstico, se ingresa a CERPO, en donde se realiza una atención multidisciplinaria (medicina materno-fetal, cirugía y cardiología pediátricas, psicología, neonatología y genética clínica). Asimismo, se solicita el estudio complementario según corresponda (ecocardiografía, neurosonografia, resonancia magnética fetal, cariograma fetal, función renal y estudio de infección transplacentaria). Cabe destacar que todas las pacientes incluidas en esta base de datos, o un adulto responsable, firmaron al ingreso un consentimiento informado aprobado por el Comité de Ética del Centro de Referencia de Salud Cordillera Oriente. El seguimiento posnatal se realiza telefónicamente para actualizar la información disponible en la base de datos.

Para la búsqueda se incluyeron todos los casos ingresados en esta unidad entre los años 2003 y 2019, y se seleccionaron pacientes con diagnóstico principal de «anomalía nefrourológica».

Las variables incluidas fueron edad materna, ciudad de derivación, paridad, antecedentes mórbidos, edad gestacional al ingreso, diagnóstico nefrourológico, anomalías asociadas, procedimiento invasivo (ya fuera terapéutico o diagnóstico), resultado del cariograma convencional, edad gestacional al parto, mortalidad (fetal, neonatal y posneonatal) y sobrevida al año. En relación con los distintos diagnósticos posibles, para 
esta revisión se agruparon usando la clasificación CAKUT (Tabla 1).

Los criterios de exclusión fueron fetos con pielectasia $<10 \mathrm{~mm}$ durante toda la gestación o que evolucionaron con regresión y hallazgos ecográficos sospechosos de patología renal, que se descartaron mediante ecografía o resonancia magnética fetal.

Los datos se tabularon utilizando una plantilla Excel $\AA^{\circledR}$, con datos anónimos y codificados, que se exportaron para su análisis estadístico al software STATA versión 12.1. Se determinaron medidas de tendencia central y de frecuencia. Se definió como estadísticamente significativo un valor $p<0.05$, con intervalo de confianza del $95 \%$.

\section{Resultados}

El total de pacientes ingresadas a CERPO entre el 1 de abril de 2003 y el 31 de diciembre de 2019 fue de 2509, y de ellas, 306 tuvieron diagnóstico prenatal de anomalías nefrourológicas (12.2\%). Se excluyeron 29 pacientes con pielectasia $<10 \mathrm{~mm}$ durante la gestación o con regresión, y cuatro pacientes en las que no se confirmó el diagnóstico inicial, correspondiendo finalmente la muestra a 273 pacientes $(11 \%$ de las pacientes ingresadas).

La edad gestacional promedio en el momento de ingreso a CERPO fue de 29 semanas +2 días $(14+0$ a $39+3)$. Respecto a los datos demográficos, el origen de la derivación correspondió a la Región Metropolitana en un $89 \%$ y a otras regiones del país en un $11 \%$. El $51 \%$ de las pacientes tenían entre 25 y 35 años, y el $12 \%$ correspondían a edades extremas $(<18$ y $>40$ años). El $74 \%$ de las madres no tenían antecedentes mórbidos y el $26 \%$ tenían antecedente de patología médica, siendo la más frecuente diabetes mellitus (7\%).

En cuanto a la patología nefrourológica (Tabla 2), un $69 \%$ presentó anomalías del tracto de salida, un $25 \%$ alteración del tamaño y de la forma de los riñones, un $8 \%$ alteración del número, un $2 \%$ alteración de la posición del riñón y un 3\% otra patología, fundamentalmente quiste renal. Hay que considerar que un feto puede presentar más de un tipo de alteración nefrourológica; en la tabla 3 se presentan otros sistemas comprometidos. Un 40\% presentaron alguna anomalía extrarrenal asociada, siendo las cardiopatías congénitas las más frecuentes (19).

Cuarenta y siete pacientes se consideraron de riesgo alto de aneuploidía, por la asociación con otras anomalías o por marcadores ecográficos sugerentes. A este grupo se les ofreció estudio invasivo, ya fuera
Tabla 2. Distribución de patología según clasificación cakut

\begin{tabular}{l|c|}
\hline Tijpo de anomalía & N $(\%)$ \\
\hline$N^{\circ}$ de riñones & $23(8)$ \\
\hline Tamaño y morfología renal & $68(25)$ \\
\hline Posición renal & $5(2)$ \\
Anomalías del tracto de salida & $188(69)$ \\
\hline Otros (Quiste renal) & $7(3)$ \\
\hline
\end{tabular}

Tabla 3. Anomalias extra renales asociadas

\begin{tabular}{lc} 
Tipo de anomalía & $\mathbb{N}(\%)$ \\
\hline Liquido amniotico disminuido & $51(34)$ \\
Cardiopatía & $53(19)$ \\
SNC & $16(9)$ \\
Gastrointestinal & $8(3)$ \\
Cara & $7(3)$ \\
RCF & $23(8)$ \\
Total & 107
\end{tabular}

SNC: sistema nervioso centra; RCF: restricción de crecimiento fetal

mediante biopsia de vellosidades coriales, amniocentesis o cordocentesis, dependiendo de la edad gestacional. Treinta y ocho pacientes se sometieron a un procedimiento invasivo, mientras que nueve declinaron dicho estudio. Las alteraciones cromosómicas se presentaron en un $34 \%$ de las pacientes estudiadas, principalmente las trisomías 18 (17\%) y 13 (6\%) (Tabla 4).

Se realizaron procedimientos invasivos terapéuticos en 19 pacientes. En 15 con diagnóstico de megavejiga se realizó vesicocentesis y en cinco se realizó un shunt vesicoamniótico. La vesicocentesis diagnóstica se realizó en caso de sospecha de obstrucción del tracto urinario inferior sin otra anomalía asociada de alto riesgo vital, para determinar la función renal prenatal y seleccionar aquellos que se beneficiarían del shunt vesicoamniótico. En cinco pacientes se realizó amnioinfusión por oligohidroamnios (OHA) grave asociado a displasia renal bilateral en tres casos y a megavejiga en dos.

La edad gestacional promedio en el momento del parto fue de 37 semanas. El 56\% fueron partos vaginales y el $44 \%$ fueron cesáreas. La sobrevida global en los casos de patología nefrourológica fue del $63 \%$ 
Tabla 4. Anomalias cromosomicas

\begin{tabular}{l|c|}
\hline Trisomia 18 & N $(\%)$ \\
\hline Trisomia 13 & $2(17)$ \\
Trisomia 21 & $1(3)$ \\
Turner $(45, \mathrm{X0})$ & $1(3)$ \\
Tetraploidia & $1(3)$ \\
Cariograma normal & $25(69)$ \\
Sin mitosis (no evaluable) & 2
\end{tabular}

al año. La mortalidad fetal fue del $7 \%$, la mortalidad neonatal fue del $16 \%$ y 31 pacientes se perdieron en el seguimiento prenatal (2) o posnatal (29). La sobrevida al año de los pacientes con patología nefrourológica se relacionó directamente con la edad gestacional de derivación a CERPO: los que no sobrevivieron al año ingresaron a CERPO a una edad gestacional significativamente menor que aquellos con sobrevida al año (24 vs. 30 semanas; $p<0.0001$ ). Sin embargo, la edad gestacional de ingreso promedio para todos los pacientes con patología nefrourológica fue de 29 semanas +2 días, dada su buena sobrevida global.

Por otro lado, la sobrevida al año fue significativamente menor en los pacientes derivados de regiones en comparación con los de la región metropolitana ( $23 \%$ vs. $77 \%$; $p<0.0001)$.

Se analizó la sobrevida por grupo según la clasificación CAKUT y se observó una sobrevida al año del $22 \%$ para las anomalías del número de riñones, del $46 \%$ para las anomalías de tamaño y forma de los riñones, del $60 \%$ para las anomalías de la posición renal y del $72 \%$ para las anomalías del tracto de salida (Fig. 1). La sobrevida al año de los pacientes con anomalías del tracto de salida alcanzó el $81 \%$ si se excluyen los pacientes con diagnóstico prenatal de megavejiga, comprendiendo fetos con dilatación de la vía urinaria superior (hidronefrosis e hidroureteronefrosis) sin signos de uropatía obstructiva.

Los pacientes con diagnóstico prenatal de megavejiga presentaron una sobrevida al año del $28 \%$, con una mortalidad fetal del $24 \%$, una mortalidad neonatal del $34 \%$ y una mortalidad posneonatal del $3 \%$; cuatro pacientes se perdieron en el seguimiento prenatal (1) o posnatal (3). Por otro lado, se observó que los pacientes con patología nefrourológica asociado a $\mathrm{OHA}$ (índice de líquido amniótico $<5 \mathrm{~cm}$ ) y sin evidencias

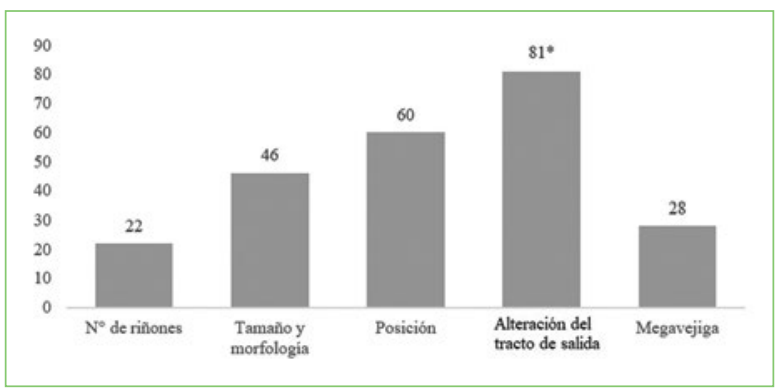

Figura 1. Sobrevida al año según la clasificación CAKUT.

de uropatía obstructiva asociada (hidroureteronefrosis bilateral o megavejiga) presentaron una sobrevida al año de solo el $3 \%$. En este grupo de 31 pacientes predominó el compromiso bilateral (94\%) con displasia o agenesia renal y enfermedad renal poliquística, con solo dos pacientes con patología unilateral: un feto con displasia renal multiquística unilateral y $\mathrm{OHA}$ grave con sobrevida al año y un feto con agenesia renal derecha cuya gestación se interrumpió en la semana 21 (no viable). Ninguno de los fetos que presentaron anhidramnios $(74 \%)$ sobrevivió más allá del periodo neonatal.

Se observaron signos ecográficos de hipoplasia pulmonar en el $45 \%$ de los casos, considerando como tales la circunferencia torácica menor del percentil 5 , o una relación entre área torácica y área cardiaca menor del percentil 5, o una relación entre la circunferencia torácica y la circunferencia abdominal menor de 0.6. El diagnóstico de hipoplasia pulmonar por resonancia magnética fetal se realizó a partir de la estimación del volumen pulmonar.

\section{Discusión}

La patología nefrourológica constituye una causa frecuente de derivación para evaluación en CERPO, siendo principalmente pacientes del área metropolitana. La gran mayoría de estas alteraciones consisten en condiciones unilaterales con un buen pronóstico desde el punto de vista de función renal posnatal, y pueden manejarse en su hospital de origen. Por esta misma razón, los pacientes ingresados a CERPO derivados de otras regiones eran significativamente de peor pronóstico que los del área metropolitana con mayor concentración de patología perinatal compleja. En esta serie, los casos ingresados precozmente fueron los más graves, tales como fetos con megavejiga 
o patología renal asociada a disminución del volumen de líquido amniótico.

También destaca en esta cohorte que el $18 \%$ de los casos se asociaron con anomalías de otros sistemas, siendo las cardiacas las más frecuentes. El volumen de líquido amniótico es reflejo de la función renal desde las 16 semanas de gestación ${ }^{14}$, lo que explica que esta sea una de las anomalías que más se asoció a la patología nefrourológica. Es importante destacar la importancia de un examen detallado de la anatomía fetal y la ecocardiografía en todos los casos, por la frecuente asociación con anomalías extrarrenales. En cuanto a la sobrevida global al año, alcanzó un 63\%, lo cual se correlaciona con el principal grupo CAKUT en esta serie, en este caso las anomalías del tracto de salida (69\%), con una sobrevida al año del $72 \%$. Cabe destacar que en la presente serie se incluyeron en este grupo solo pacientes con dilataciones de la vía urinaria moderadas o graves, excluyendo hidronefrosis < $10 \mathrm{~mm}$ de diámetro de pelvis anteroposterior (AP) o pacientes en quienes se descartó patología nefrourológica en su evolución. Un subgrupo especial dentro de este grupo son los fetos con diagnóstico de megavejiga, los cuales en algunos casos fueron sometidos a procedimientos invasivos, como vesicocentesis o realización de shunt derivativo vesicoamniótico. El pronóstico en estos casos es ominoso y la sobrevida al año en nuestra serie fue del $28 \%$.

No obstante, el subgrupo con peor sobrevida según nuestros resultados fueron los fetos con diagnóstico de $\mathrm{OHA}$ de causa renal, en los que la mortalidad a los 28 días de vida fue del $97 \%$. El único paciente con sobrevida presentaba una patología renal unilateral, por lo cual tenía buen pronóstico desde el punto de vista de función renal posnatal, pero presentó OHA durante el embarazo de causa incierta. Las anomalías renales asociadas con $\mathrm{OHA}$ que pueden distinguirse antenatalmente por ultrasonido son la displasia, la hipoplasia o la agenesia renal bilateral, el riñón poliquístico autosómico recesivo y la uropatía obstructiva ${ }^{15}$. En nuestra serie excluimos de este análisis a los pacientes con signos de uropatía obstructiva, tales como megavejiga, dado que confieren mayor heterogeneidad al grupo en estudio, tienen un mejor pronóstico perinatal en relación con las displasias o agenesias renales ${ }^{17,18} \mathrm{y}$, por otro lado, pueden ser candidatos a alguna intervención prenatal. La gran mayoría de fetos con OHA de causa renal presentaron patología bilateral, y si consideramos para analizar la mortalidad solo la patología renal bilateral asociada a OHA, la mortalidad a los 28 días fue del $100 \%$. Las patologías renales bilaterales a menudo conducen a disfunción renal antenatal que se caracteriza por una disminución del débito urinario fetal y, en consecuencia, OHA con un pronóstico ominoso, especialmente porque el desarrollo pulmonar también puede verse afectado, ya que la maduración pulmonar es dependiente de la producción de líquido amniótico ${ }^{16}$. Nuestros resultados confirman lo anterior, pues ninguno de los fetos que presentaron anhidramnios (74\%) sobrevivió más allá del periodo neonatal, probablemente por la hipoplasia pulmonar asociada; sin embargo, solo se observaron signos de hipoplasia pulmonar en el $45 \%$ de los casos con OHA por ecografía o resonancia magnética fetal. Al respecto, nos parece importante informar, sobre todo en el contexto de la Ley N. 21.030 vigente desde el año 2017, que despenaliza la interrupción voluntaria del embarazo en tres causales. Dentro de la segunda causal, por patología nefrourológica solo se incluyen la agenesia renal y los riñones multiquísticos o poliquísticos, ambos asociados a hipoplasia pulmonar, secundario al anhidramnios. Considerando la experiencia en este centro en relación a la patología renal bilateral asociada a OHA y su mal pronóstico, es necesario realizar estudios con mayor número de pacientes para eventualmente poder determinar esta patología como parte de la causal 2 de la Ley de interrupción voluntaria del embarazo en tres causales.

\section{Conclusiones}

Las anomalías del sistema nefrourológico son un diagnóstico prenatal frecuente y muy variado. La mayoría se asocian con anomalías del tracto de salida, principalmente hidronefrosis. Un $40 \%$ de los casos presentan anomalías extrarrenales, reforzando la importancia de realizar un examen anatómico detallado. De forma global, los resultados perinatales muestran una sobrevida al año del $63 \%$, pero existen diferencias significativas entre los distintos tipos de patología nefrourológica según la clasificación CAKUT y su asociación con OHA e hipoplasia pulmonar. Se debe continuar el seguimiento a largo plazo para determinar la evolución de la función renal según cada diagnóstico o hallazgo prenatal.

\section{Financiamiento}

Los autores no recibieron financiamiento en este trabajo. 


\section{Conflicto de intereses}

Los autores no tienen un conflicto de intereses en este trabajo.

\section{Responsabilidades éticas}

Protección de personas y animales. Los autores declaran que para esta investigación no se han realizado experimentos en seres humanos ni en animales.

Confidencialidad de los datos. Los autores declaran que han seguido los protocolos de su centro de trabajo sobre la publicación de datos de pacientes.

Derecho a la privacidad y consentimiento informado. Los autores han obtenido el consentimiento informado de los pacientes y/o sujetos referidos en el artículo. Este documento obra en poder del autor de correspondencia.

\section{Bibliografía}

1. Murugapoopathy V, Gupta IR. A primer on congenital anomalies of the kidneys and urinary tracts (CAKUT). Clin J Am Soc Nephrol. 2020;15:723-31.

2. Queißer-Luft A, Stolz G, Wiesel A, Schlaefer K, Spranger J. Malformations in newborn: results based on 30940 infants and fetuses from the Mainz congenital birth defect monitoring system (1990-1998). Arch Gynecol Obstet. 2002;266:163-7.

3. Grandjean H, Larroque D, Levi S. The performance of routine ultrasonographic screening of pregnancies in the Eurofetus Study. Am J Obstet Gynecol. 1999;181:446-54

4. Bhide A, Sairam S, Farrugia MK, Boddy SA, Thilaganathan B. The sensitivity of antenatal ultrasound for predicting renal tract surgery in early childhood. Ultrasound Obstet Gynecol. 2005;25:489-92.
5. Yulia A, Winyard P. Early human development management of antenatally detected kidney malformations. Early Hum Dev. 2018;126:38-46.

6. Sanna-Cherchi S, Ravani P, Corbani V, Parodi S, Haupt R, Piaggio G, et al. Renal outcome in patients with congenital anomalies of the kidney and urinary tract. Kidney Int. 2009;76:528-33.

7. Winyard P, Chitty LS. Dysplastic kidneys. Semin Fetal Neonatal Med. 2008;13:142-51.

8. Lewis M, Shaw J, Reid C, Evans J, Webb N, Verrier-Jones K. Demography and management of childhood established renal failure in the UK (Chapter 13). Nephrol Dial Transplant. 2007;22 (Suppl 7):vii165-75.

9. Caiulo VA, Caiulo S, Gargasole C, Chiriacò G, Latini G, Cataldi L, et al. Ultrasound mass screening for congenital anomalies of the kidney and urinary tract. Pediatr Nephrol. 2012;27:949-53.

10. Andrés-Jensen L, Jørgensen FS, Thorup J, Flachs J, Madsen JL, Maroun $\mathrm{LL}$, et al. The outcome of antenatal ultrasound diagnosed anomalies of the kidney and urinary tract in a large Danish birth cohort. Arch Dis Child. 2016;101:819-24.

11. Wiesel A, Queisser-Luft A, Clementi M, Bianca S, Stoll C; EUROSCAN Study Group. Prenatal detection of congenital renal malformations by fetal ultrasonographic examination: an analysis of 709,030 births in 12 European countries. Eur J Med Genet. 2005;48:131-44.

12. Deshpande C, Hennekam RCM. Genetic syndromes and prenatally detected renal anomalies. Semin Fetal Neonatal Med. 2008;13: 171-80.

13. Nazer J, Cifuentes L, Ramírez C. Malformaciones urinarias del recién nacido. Estudio ECLAMC 1998-2010. Rev Chil Pediatr. 2011;82: $512-9$.

14. Magann EF, Sandlin AT, Ounpraseuth ST. Amniotic fluid and the clinical relevance of the sonographically estimated amniotic fluid volume: oligohydramnios. J Ultrasound Med. 2011;30:1573-85.

15. Aulbert W, Kemper MJ. Severe antenatally diagnosed renal disorders: background, prognosis and practical approach. Pediatr Nephrol. 2016:31:563-74.

16. Grijseels E, Van Hornstra P, Govaerts L. Outcome of pregnancies complicated by oligohydramnios or anhydramnios of renal origin. Prenat Diagn. 2011;31:1039-45.

17. Mehler K, Gottschalk I, Burgmaier K, Volland R, Büscher AK, Feldkötter $\mathrm{M}$, et al. Prenatal parental decision-making and postnatal outcome in renal oligohydramnios. Pediatr Nephrol. 2018;33:651-9.

18. Spiro JE, Konrad M, Rieger-Fackeldey E, Masjosthusmann K, Amler S, Klockenbusch W, et al. Renal oligo- and anhydramnios: cause, course and outcome - a single-center study. Arch Gynecol Obstet. 2015;292:327-36. 